\section{What makes popcorn pop}

SIR - Corn and popcorn belong to the same plant species Zea mays mays ${ }^{1}$, but only popcorn pops. Previous studies on this question have been restricted to popcorn fruits and have demonstrated that pericarp (hull) and starch are important for popping 2,3

We compared four distinct varieties of corn, $C 10, C 12, C 16$ and $C 08$, and three popcorn varieties, $P 60, P 61$ and $P 62$. We measured thermal diffusivity of the pericarp using a photoacoustic technique ${ }^{4}$. Popping values, the ratios of expanded to original volumes, were $28.3,32.2$ and 33.5 in $P 62, P 61$ and $P 60$, respectively. In the corn varieties, popping values were $1.7,3.9,4.4$ and 8.7 , respectively, for $C 12, C 08, C 10$ and $C 16$. These results indicate a clear difference in popping ability between popcorn and corn types. We found highly negative simple Pearson correlations between expansion capacity and kernel weight $(r=-0.91)$, kernel volume $(r=-0.91)$ and proportion of opaque/brilliant endosperm $(r=-0.77)$. We also found a highly positive correlation between expansion capacity and pericarp thickness $(r=0.81)$, and positive correlations between the proportion of opaque/brilliant endosperm and kernel weight $(r=0.88)$ and volume $(r=0.89)$. These findings indicate the importance of both the pericarp thickness and the endosperm starch

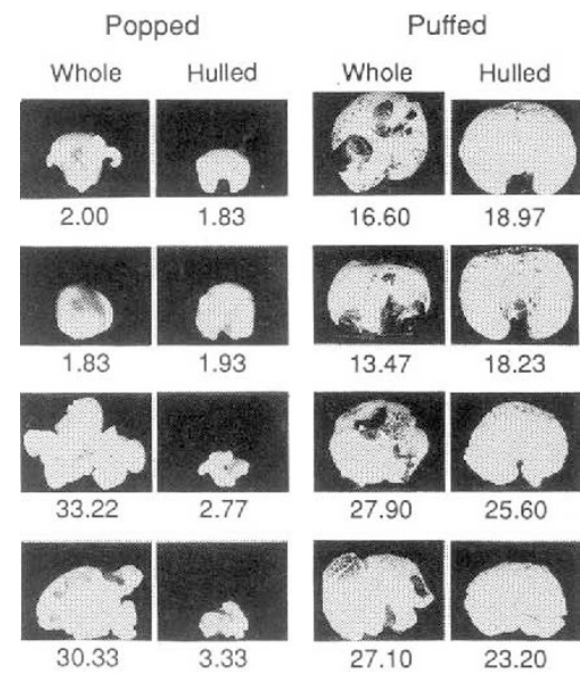

Kernel expansion in (from top row to bottom row) two varieties of corn, $C 10$ and $C 12$, and two of popcorn, $P 61$ and $P 62$, subjected to popping and puffing with whole and hulled kernels. Numbers indicate pop expansion, the ratio of the expanded to the original volume. For the popping process, a sample of $30 \mathrm{~g}$ of seeds was put in corn oil at a temperature of around $180^{\circ} \mathrm{C}$. Puffing was done by placing a $160-g$ sample of kernels in a rotatory pressure chamber (Canon) heated by a propane flame at $180^{\circ} \mathrm{C}$ with an internal pressure of 135 p.s.i. with corn. type to popping quality.

Popcorn pericarp has a thermal diffusivity and a thermal conductivity on average 2.9 and 2.0 times higher, respectively, than those of the corn pericarp. We found a high positive correlation between the thermal diffusivity and the expansion capacity $(r=0.94)$. The higher values of the thermal diffusivity and conductivity suggest that the popcorn pericarp is less amorphous, that is, more structurally organized, than the corn pericarp.

Birefringence measurements of the pericarp (expressed as optical path difference) gave an average value of 56.7 $\mathrm{nm}$ for popcorn and $38.1 \mathrm{~nm}$ for corn pericarp $(t=7.42$, that is, significantly different at the $0.1 \%$ level) in the less birefringent sectors (brilliant). The most birefringent areas displayed values of $93.6 \mathrm{~nm}$ for popcorn and $64.8 \mathrm{~nm}$ for corn pericarp $(t=12.81)$, indicating a more crystalline arrangement of cellulose and a higher degree of fibrillar packing in popcorn pericarp compared

We carried out popping and puffing experiments to investigate the contribution of pericarp and type of endosperm starch to popping characteristics (see figure). Pericarp extraction in popcorn varieties reduced popping by $90.4 \%$, confirming earlier studies ${ }^{2}$. In hulled kernels, popcorn starch was $62.2 \%$ more suitable for popping than was that of corn endosperm. Thus the pericarp characteristics, rather than those of the endosperm, seem to be most important for popping.

The puffing experiment showed that the starch quality of corn kernels also has the potential to produce good expansion ability, for in this case, the metal cap of the pressure chamber replaced the weak corn pericarp. Assuming a pressure vessel model ${ }^{2,5,6}$ for the popping mechanism, where the pericarp acts as a vessel cap, we can draw two conclusions. First, using our thermal data, the popcorn pericarp heat-transfer coefficient is roughly 1.9 times that of corn. This implies a more efficient heat transfer into the popcorn kernel, so that its inner temperature grows faster and higher than that of corn pericarp, while the endosperm temperature stays lower. The lower temperature of the popcorn pericarp minimizes the chances of its combustion when the starch granules are at a temperature producing maximum expansion. On the other hand, the corn pericarp becomes dark and calcined at the temperature of maximum starch expansion. Second, the higher mechanical resistance of popcorn pericarp (approximately four times that of corn pericarp) means that it can sustain higher pressure, favouring high popping in popcorn varieties. The thermal and mechanical properties of the pericarp show that the pericarp acts as a cap.

W. J. da Silva

B. C. Vidal

M. E. Q. Martins

Instituto de Biologia,

Universidade Estadual de Campinas,

Caixa Postal 6109,

13081-970 Campinas,

Sao Paulo, Brazil

H. Vargas

A. C. Pereira

M. Zerbetto

Instituto de Física,

Universidade Estadual de Campinas,

Caixa Postal 6165,

13081-970 Campinas,

Sao Paulo, Brazil

Lulz C. M. Miranda

Laboratório Associado de Sensores e Materiais,

Instituto de Pesquisas Espaciais,

Caixa Postal 515, 12201-970 São José

dos Campos,

Sao Paulo, Brazil

1. Doebley, J. F. \& Iltis, H. H. Am. J. Bot. 67, 982-993 (1980).

2. Hoseney, R. C., Zelesnak, K. \& Abdelrahman, A. J. Cereal Sci. 1. 43-52 (1983)

3. Haugh, C. G., Lien, R. M., Hanes, R. E. \& Ashman, R. B. Trans. Am. Soc. Agric. Engng 176, 168-171 (1976).

4. Vargas, H. \& Miranda, L. C. M. Phys. Rep. 161, 43-101 (1988).

5. Strehlow; R. A. Combustion Fundamentals (McGraw-Hill, New York, 1984).

6. Semenov, N. N. Chemical Kinetics and Chain Reactions (Oxford Univ. Press, 1935)

\section{Sperm costs and lifespan}

SIR - Van Voorhies reported in his study on the nematode Caenorhabditis elegans $^{1}$ that sex reduces the lifespan of males, but not of hermaphrodites. Because hermaphrodites do not produce any sperm during mating (in contrast to males), he assumed that spermatogenesis is the constraining factor in reproduction. To test this, he used mutants with deficient spermatogenesis. With them, he claims to have shown that the production of sperm rather than the physical act of mating has a significant impact on male lifespan.

If this were true, one would also expect to find an elongated lifespan of unmated mutant males compared to unmated wild-type males (which produce more sperm). However, the data presented in Figs 2 and 4 of Van Voorhies's paper suggest that there is no difference in the lifespan of unmated wild-type (Fig. 2) and unmated mutant males (Fig. 4). Even if these two figures represent two different sets of experiments, the conditions under which they were done 